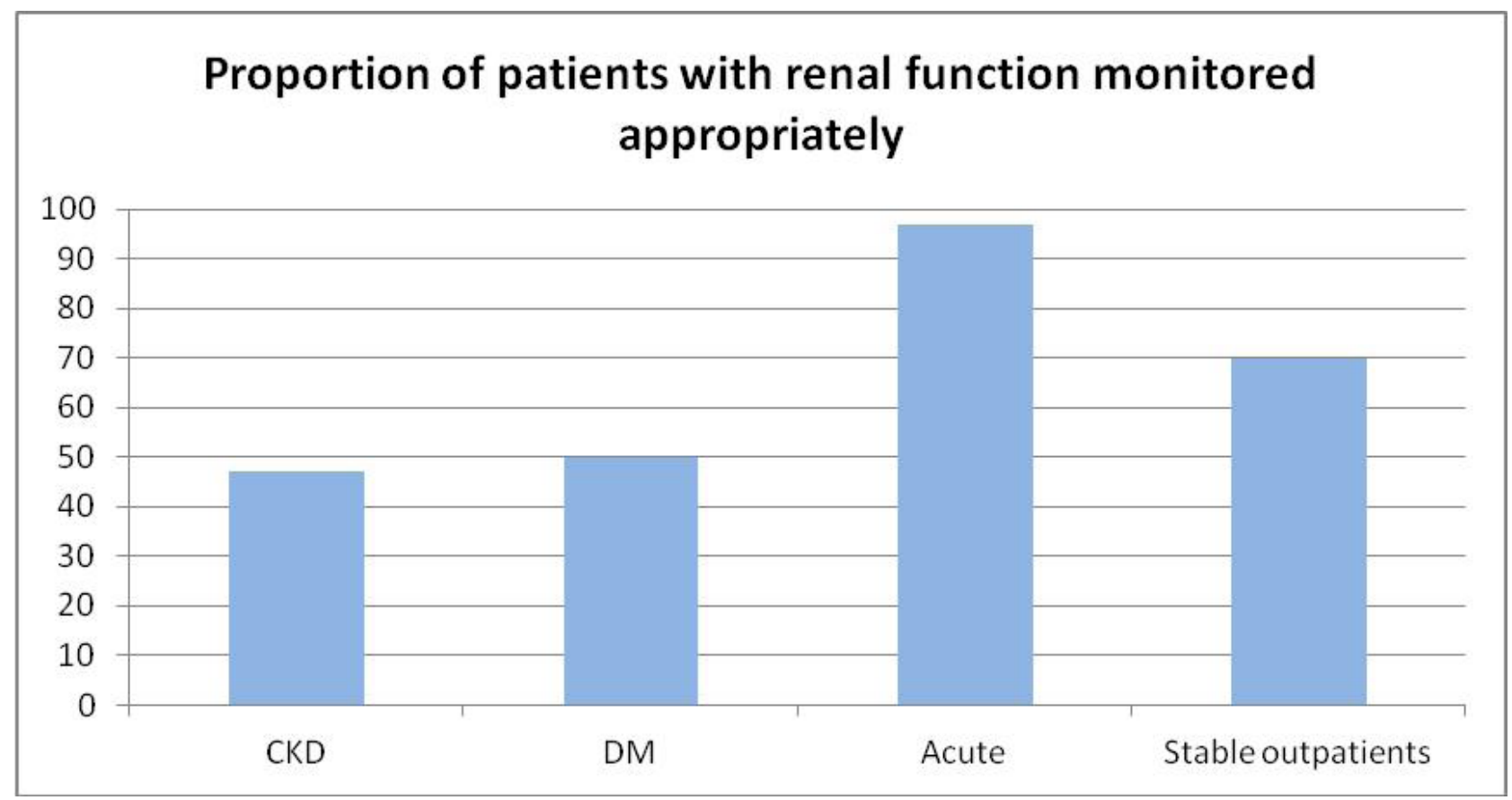

Abstract P66 Figure 1

CT scans. The majority of departments scanned the entire lung (62\%), 20\% used limited slices and 20\% used a combination as part of follow up. Only 15\% of departments used lung nodule volume measurements routinely, with a further $20 \%$ having access on request.

Conclusions There is significant variation both in the way patient's are followed-up as well as the methods of scanning deployed. Some trusts have developed streamlined pathways to monitor patients, without using valuable clinic slots. The chest physician is very much reliant on the organisation and expertise of their radiology department, with a significant majority not having access to low dose CT or lung nodule volumes. It is a crucially important area that requires continued improvement, both in achieving earlier cancer detection, balanced against the need for limiting the radiation dose.

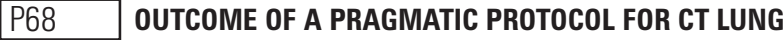 NODULE SURVEILLANCE IN A UK DISTRICT GENERAL HOSPITAL}

doi:10.1136/thoraxjnl-2012-202678.209

MT Reichmann, D Edwards, A Sivaloganathan, A Drury, D Laws. Royal Bournemouth Hospital, Bournemouth, United Kingdom

Introduction and objectives The appropriate way to follow-up indeterminate pulmonary nodules found incidentally on CT scanning has caused clinicians and radiologists in the UK some concern. Guidelines developed by the Fleischner Society in 2005 were based on studies outside of the UK. Our hospital developed local guidance for lung nodule surveillance prior to the publication of Fleischner guidelines which were designed to be pragmatic and easy to follow. We present the results of our experience.

Methods Outcomes of patients undergoing the local lung nodule surveillance programme in our hospital from 2004 to 2011 were analysed. Eligibility criteria included initial lung nodules 5-10 mm diameter; previous or current smokers; aged 45-75 years old with good performance status. Those with 5 or more nodules more than $5 \mathrm{~mm}$ diameter, benign calcification, or patients already under follow up, e.g. oncology patients, were excluded. A stamp was placed in the notes and on CT request forms to record and remind clinicians of the criteria. In accordance to our protocol CT scans were performed at 6, 18 and 30 months from the index scan.

Results 107 patients were followed up but only 63 patients fulfilled the initial inclusion criteria. This shows that despite a pragmatic protocol, clinicians will often interpret it differently when faced with an individual. The commonest reason was nodule size over $10 \mathrm{~mm}$. Of those eligible, the outcomes were recorded as to whether nodule confirmed as cancer (positive), nodule size reduced or unchanged over 30 months (negative), surveillance cut short as a clinical decision and those still under surveillance.

Of the 63 patients, 2 were found to have lung cancer (see Figure 1). Of those patients who were not eligible, but still underwent the surveillance programme, 6 were found to have cancer. These were not eligible because nodule size was over $10 \mathrm{~mm}$.

Conclusions Our study shows that a simple protocol is helpful to clinicians, but will be adapted according to the clinicians' belief. In our study $3 \%$ of nodules $5-10 \mathrm{~mm}$ were early cancers. Nodules over $10 \mathrm{~mm}$, which were bigger than our criteria but followed up within this protocol, were more likely to be cancerous $(14 \%)$

\section{P69 DO PATIENTS PROGRESS WHILST UNDERGOING DIAGNOSIS AND STAGING FOR LUNG CANCER: A RETROSPECTIVE AUDIT?}

doi:10.1136/thoraxjnl-2012-202678.210

${ }^{1}$ R Kwong, ${ }^{1} \mathrm{C}$ Bradshaw, ${ }^{2} \mathrm{~L}$ Finney, ${ }^{3} \mathrm{GJ}$ Burkill, ${ }^{2} \mathrm{SR}$ Doffman. ${ }^{1}$ Brighton Sussex Medical School, Brighton, East Sussex; '2Department of Respiratory Medicine, Brighton and Sussex University Hospitals NHS Trust, Brighton, East Sussex: ${ }^{3}$ Department of Radiology, Brighton and Sussex University Hospitals NHS Trust, Brighton, East Sussex

Background In the last 10 years, the survival rate in lung cancer in the UK has improved, but remains lower than some counterpart 